\title{
Systemic pseudohypoaldosteronism-1 with episodic dyslipidemia in a Sudanese child
} \author{
Mohamed Abdullah ${ }^{6}$ \\ 1Department of Pediatric Endocrinologist, Gaafar Ibn Auf Children's Tertiary Hospital, Khartoum, Sudan, 2Department \\ of Pediatrics, Prince Sattam Bin Abdulaziz University, Alkharj, Kingdom of Saudi Arabia, ${ }^{3}$ Department of Pediatric \\ Respirologist, Elswaidi Charity Hospital, Khartoum, Sudan, ${ }^{4}$ Department of Pediatrics, University of Shendi, Shendi, \\ Sudan, ${ }^{5}$ Clinical Lab Operations at Centogene Laboratory, Rostock, Mecklenburg-Vorpommern, Germany, and \\ ${ }^{6}$ Departmentof Pediatric Endocrinology, Faculty of Medicine, University of Khartoum, Khartoum, Sudan
}

\section{Summary}

Systemic pseudohypoaldosteronism type 1 (PHA1) is a rare genetic syndrome of tissue unresponsiveness to aldosterone caused by mutations affecting the epithelial $\mathrm{Na}$ channel $(\mathrm{ENaC})$. The classical presentation is life-threatening neonatal/ infantile salt-losing crises that mimic congenital adrenal hyperplasia (CAH). Consistently, extra-renal manifestations, including respiratory symptoms that resemble cystic fibrosis, are well reported. Clinical diagnosis is made by the presence of hyponatremia, hyperkalemia, metabolic acidosis, respiratory symptoms, evidence of high renal and extra-renal salt loss in addition to high plasma renin and aldosterone levels. We herein report a novel manifestation of PHA1: episodic dyslipidemia in a 7-month-old Sudanese boy that occurred during the salt-losing crises. Whole exome sequencing of the patient revealed one homozygous missense variant c.1636G>A p.(Asp546Asn) in the SCNN1B gene, confirming our clinical and laboratory findings that were compatible with PHA1. This report aims to highlight the possible explanation of dyslipidemia in PHA1 and its expected consequences in the long term.

\section{Learning points:}

- A child presenting with features that mimic salt-losing congenital adrenal hyperplasia (CAH) crises that do not respond to glucocorticoid and mineralocorticoid therapy should alert the pediatricians to the possibility of endorgan resistance to aldosterone.

- Pseudohypoaldosteronism type 1 (PHA1) can be diagnosed even in the absence of advanced laboratory investigations.

- To our knowledge, this is the first case of systemic PHA1 to have a documented episodic dyslipidemia (primarily as marked hypertriglyceridemia).

\section{Background}

Pseudohypoaldosteronism type 1 (PHA1) is a rare syndrome of tissue unresponsiveness to aldosterone action. The condition has two clinically and genetically distinct forms. The autosomal dominant renal PHA1 is due to a mutation in the gene encoding the mineralocorticoid receptors (1). Familial autosomal dominant renal PHA1 in up to $87 \%$ of kindreds and sporadic renal presentation in
$81 \%$ of patients are due to mutations in the gene NR3C2. The rarer systemic form of PHA1 results from an autosomal recessive mutation in the genes encoding the epithelial sodium channel $(\mathrm{ENaC}) \alpha, \beta$, or $\gamma$ subunits, which are expressed in the epithelial lining of several tissues, for example, distal renal tubules, lungs, sweat glands, and distal colon (1). Clinical presentation of the systemic 
form is uniformly characterized by repeated neonatal and infantile salt-wasting crises with severe dehydration, hyponatremia, hyperkalemia, and metabolic acidosis. This salt-and-fluid-losing and potassium- and protonretaining pathophysiology are believed to persist lifelong in most of the cases $(2,3)$. Also, extra-renal manifestations are constant features of the disease. Failure to thrive is consistently reported in systemic PHA1 (4). Patients have pulmonary manifestations resembling cystic fibrosis of variable severity and age of onset (5). Patients with PHA1 were found to have double the volume of airway surface liquid compared to healthy children (5). The liquid impairs mucociliary clearance and narrows airways lumen and/ or dilutes surface-active materials. As the patient grows, respiratory symptoms improve unless irreversible changes in airways structure take place (5). Dermal eruptions and infections have been frequently reported with PHA1 and are usually described as miliaria rubra-like lesions.

The diagnosis of Systemic PHA1 requires the demonstration of particularly high plasma renin or renin activity (PRA), as well as markedly elevated aldosterone, in addition to normal glucocorticoid axis and normal adrenal steroids in the context of a salt-losing event. Causes of a secondary transient form of PHA1, such as urinary tract infections, should be ruled out (6). Demonstration of high sweat sodium and chloride level will differentiate systemic from renal PHA1. It is always useful to genetically confirm the clinical diagnosis in cases of hypo- or pseudohypoaldosteronism, as rare causes of hypoaldosteronism may be identified, that is, heterozygous mutations in the cholesterol side-chain cleavage enzyme gene (CYP11A1), and probably absolutely necessary in cases that are unresponsive to mineralocorticoid replacement therapy, which - together with salt replacement - is the preferred approach of pediatric endocrinologists even in NR32C2 mutations, even if theoretically there would be an expected resistance to their action. However, our understanding of $\mathrm{NaCl}$ handling and regulation in the collecting duct is moving steadily forward toward a more comprehensive model. In this report, we are describing the clinical course and diagnostic process of a Sudanese child with systemic PHA1, including recurrent episodes of transient hyperlipidemia, which we believe has not been previously reported in the literature.

\section{Case presentation}

A 7.5-month-old Sudanese boy was admitted to our pediatric endocrinology unit for evaluation of two episodes of dehydration with hyponatremia and hyperkalemia with suspicion of congenital adrenal hyperplasia (CAH). He was a full-term infant born to healthy consanguineous parents - no family history of neonatal deaths or endocrine illnesses.

The first symptoms were reported at the age of 2 weeks with fever and increased breathing effort that was treated as pneumonia. Since then, the patient had repeated lung infections that were treated with i.v. antibiotics and bronchodilators and were characterized by moist cough, labored breathing, wheezing, and sometimes fever. At 7 months of age, he developed vomiting, diarrhea, and hyponatremic dehydration associated with hyperkalemia in addition to the chest symptoms. On admission to our center, he was conscious, thin, and moderately dehydrated. Blood pressure was normal. He had acidotic breathing with scattered wheezes and squeaks bilaterally. There was no digital clubbing, and he did not have apparent dysmorphic features. He had normal male genitalia. There was no hyperpigmentation on mucous membranes nor over the circumcision scar. There was a hyperpigmented papular skin rash in between the digits, axilla, and over the trunk with scratch marks that appeared just prior to his recent illness. The patient's weight was $5 \mathrm{~kg}$ ( $<3 \mathrm{rd}$ percentile), length: $64 \mathrm{~cm}$ (3rd percentile), and head circumference: 41 $\mathrm{cm}$ (10th percentile). Other system examination findings were unremarkable.

\section{Investigation}

Initial biochemistry showed hyponatremia, hyperkalemia, and metabolic acidosis (Table 1). As CAH was the first differential diagnosis, samples were drawn for serum cortisol, ACTH, and 17-hydroxyprogesterone, all of which turned up to be normal but with remarkably high serum renin and aldosterone levels (Table 1). The renal and adrenal ultrasonography scan was normal. A chest x-ray showed bilateral hyperinflation.

Blood samples were noticed to be lipemic, so a fasting blood sample for lipid profile was examined that revealed a high cholesterol level and markedly high triglycerides (TG) with low high-density lipoprotein (HDL) representing combined dyslipidemia (Table 2). This finding was noticed henceforward whenever the patient was presented with bouts of hyponatremic dehydration and hyperkalemia. In these instances, blood samples for lipid profile were mainly taken on the following days of admission, when the patient was still hyperkalemic but reasonably rehydrated. The lipid profile was completely normal in between the attacks. 
Table 1 Initial laboratory tests.

\begin{tabular}{l}
\hline Test \\
\hline S. $\mathrm{Na}^{+}$ \\
S. $\mathrm{K}^{+}$ \\
S. Cl- \\
Blood sugar \\
Blood urea nitrogen \\
Serum creatinine \\
C-reactive protein \\
Blood culture \\
Complete blood picture \\
Liver function test \\
Blood gas \\
Serum cortisol \\
ACTH \\
Serum 17OHP* \\
S. aldosterone \\
S. renin \\
Sweat test** \\
\end{tabular}

\begin{tabular}{l} 
Result (reference range) \\
\hline $109(135-145 \mathrm{mEq} / \mathrm{L})$ \\
$7.9(3.5-5 \mathrm{mEq} / \mathrm{L})$ \\
$98(98-106 \mathrm{mEq} / \mathrm{L})$ \\
$6.7(4.4-10 \mathrm{mmol} / \mathrm{L})$ \\
$9.1(0-3.3 \mathrm{mmol} / \mathrm{L})$ \\
$0.03(0.02-0.06 \mathrm{mmol} / \mathrm{L})$ \\
Negative \\
Negative \\
Microcytic hypochromic anemia \\
Normal \\
$\mathrm{pH}=7.2(7.35-7.45), \mathrm{HCO} 3=10 \mathrm{mmol} / \mathrm{L}(18-22), \mathrm{PCO}_{2}=26 \mathrm{mmHg}(25-40)$ \\
$538(171-535 \mathrm{nmol} / \mathrm{L})$ \\
$0.4(0.35-3 \mathrm{pmol} / \mathrm{L})$ \\
$1.8(0.07-1.7 \mathrm{nmol} / \mathrm{L})$ \\
$2775(111-860 \mathrm{pmol} / \mathrm{L})$ \\
$4.2(0.1-0.75 \mathrm{pmol} / \mathrm{L})$ \\
Sweat for chloride $=112.66 \mathrm{mmol} / \mathrm{L}(10-65)$ \\
Sweat for sodium $=179.27 \mathrm{mmol} / \mathrm{L}(10-70)$
\end{tabular}

*170HP; 17-hydroxyprogesterone; **The test was done when the patient was stable.

\section{Treatment}

At initial admission, the patient was rehydrated with i.v. dextrose normal saline. i.v. hydrocortisone, and oral fludrocortisone were started after samples were drawn and stopped after the results. Hyperkalemia was managed with nebulized salbutamol, bicarbonate, and calcium gluconate infusion with ECG monitoring. Both sodium and potassium levels were only appropriately corrected after adding oral

Table 2 Serial fasting lipid profile during crises and in between.

\begin{tabular}{|c|c|c|c|c|}
\hline \multirow[b]{2}{*}{ Fasting lipid profile } & \multicolumn{4}{|c|}{ Age/year } \\
\hline & $\begin{array}{l}8 \text { months } \\
\text { (2016) } \\
\text { during } \\
\text { crisis* }\end{array}$ & $\begin{array}{c}1 \text { year } \\
(2016) \\
\text { follow up } \\
\end{array}$ & $\begin{array}{l}3 \text { years } \\
(2018) \\
\text { during } \\
\text { crisis* }\end{array}$ & $\begin{array}{c}4 \text { years } \\
\text { (2019) } \\
\text { follow up }\end{array}$ \\
\hline $\begin{array}{l}\text { Cholesterol } \mathrm{mmol} / \mathrm{L} \\
(<5.2)\end{array}$ & 5.8 & 4.1 & 3 & 4.65 \\
\hline $\begin{array}{l}\mathrm{TG} \mathrm{mmol} / \mathrm{L} \\
(0.45-1.71)\end{array}$ & 4.5 & 1.2 & 5.1 & 1.44 \\
\hline $\mathrm{HDL} \mathrm{mmol/L} \mathrm{(>0.91)}$ & 1 & 1.75 & 0.1 & 1.39 \\
\hline LDL mmol/L (<3.4) & 2.7 & 1.78 & 0.59 & 2.58 \\
\hline $\begin{array}{l}\text { VLDL mmol/L (0.31- } \\
0.78)\end{array}$ & 2.1 & 0.57 & 2.31 & 0.68 \\
\hline T. cholesterol/HDL & 5.8 & 2.34 & 30 & 3.34 \\
\hline LDL/HDL & 2.7 & 1.01 & 5.9 & 1.85 \\
\hline $\begin{array}{l}\mathrm{TG} / \mathrm{HDL} \mathrm{mmol} / \mathrm{dL} \\
(<1.33)\end{array}$ & 4.5 & 0.68 & 51 & 1 \\
\hline
\end{tabular}

*It should be noted that the deranged lipid profile was detected primarily upon rehydration. 'Crisis' here denotes the whole-time interval until re-compensation.

HDL, high-density lipoprotein; LDL, low-density lipoprotein; T. cholesterol, total cholesterol; TG, triglycerides; VLDL, very low-density lipoprotein. table salt ( $1 \mathrm{~g}$ of table salt contains approximately $17 \mathrm{mmol}$ sodium) that was diluted in water or milk to provide 17 $\mathrm{mmol} / \mathrm{kg} /$ dose initially and later reduced to $10 \mathrm{mmol} / \mathrm{kg} /$ dose three times a day. Oral potassium-binding resin in the form of calcium polystyrene was added at a dose of 0.5 $\mathrm{g} / \mathrm{kg} /$ day in divided doses. He was discharged home with these medications. Doses were adjusted as required during the follow-up visits. The quantitative sweat chloride test for sodium and chloride was in the cystic fibrosis range (Table 1). Total serum immunoglobulin E (IgE) and eosinophil count were within the normal range. He was seen by the dermatologist for the skin rash, which was diagnosed as scabies and responded nicely to topical permethrin treatment.

Based on these data, the clinical diagnosis of systemic PHA1 was entertained, and subsequently, exome sequencing of DNA from the patient was performed. Evaluation of the entire exome dataset, including genes related to PHA (CUL3, HSD11B2, KLHL3, NR3C2, SCNN1A, SCNN1B, SCNN1G, WNK1, WNK4) revealed one homozygous missense variant c.1636G >A, p. (Asp546Asn) in the SCNN1B gene (NM_000336.2).

\section{Outcome and follow-up}

Since then, the patient had been maintained on oral table salt and calcium resonium $1 \mathrm{~g} / \mathrm{kg} /$ day. His respiratory manifestations were managed by bronchodilators (mainly salbutamol), physiotherapy, and antibiotics when indicated. Later, he was trained on airway clearance 
techniques using an oscillating positive expiratory pressure device (Acapella) on a daily basis. Currently, at the age of 4 years, he is not on oral salt but only craves it interruptedly during acute illnesses. He is otherwise asymptomatic with normal electrolytes and lipid profile. He is growing along the third centile in both height and weight and developing normally. He did not need further admissions.

\section{Discussion}

To our knowledge, this is the first case of systemic PHA1 to have a documented dyslipidemia and the first to be reported from within Sudan. Case reports of systemic PHA1 have been particularly scarce from low-income countries (7). The published reports have depicted several incidences of unexplained neonatal deaths in siblings of reported cases, suggesting that many children might have died undiagnosed or mistakenly diagnosed as gastroenteritis in areas with limited diagnostic facilities.

Newborns or young infants presenting with symptoms of lethargy, vomiting, dehydration with hyponatremia and hyperkalemia are commonly considered by pediatricians as CAH due to 21 hydroxylase deficiency and initially treated as such unless proved otherwise later. This is what happened to our patient. Diagnoses other than $\mathrm{CAH}$, including PHA1, were considered when the patient did not respond to the usual management of CAH. PHA1 was confirmed by the result of markedly high renin and aldosterone levels and molecular genetic testing. Secondary causes of PHA1 such as urinary tract infections and malformations were excluded. Clinically, the respiratory symptoms, diarrhea, and high sweat chloride were in favor of the systemic form of PHA1. Although this triad might suggest cystic fibrosis (CF), the presence of hyperkalemia rather than hypokalemia has virtually excluded it. Unfortunately, we faced difficulty in assessing urinary electrolytes in our patient, which would be expected to show a high sodium level. There is no sufficient explanation for the intermittent nature of respiratory exacerbations (other than superadded infections). Airway clearance techniques, like those adopted in our patient, will help to mobilize airway fluids, and the positive expiratory pressure (PEP) will prevent atelectasis and open collateral channels. Acapella is one of the modern oscillating PEP devices that are used in cystic fibrosis and other lung diseases involving suppuration or abnormal airway clearance. With these measures, together with appropriate treatment of infection and vaccination, favorable respiratory outcomes are expected.

Previous case reports consistently showed early infancy presentation with salt-wasting in patients with systemic
PHA1 $(2,3,4,8,9,10)$. In our patient, the presentation of salt-losing crises was not very early, which might imply a milder renal manifestation. Phenotype variation, including timing and severity of different systemic presentations as well as improvement of symptoms over time, maybe at least partially explained by a possible genotype-phenotype relationship (2). It has been suggested in the literature that some genotypes, for example, with missense mutations in the $\alpha \mathrm{ENaC}$ gene (SCNN1A), may be associated with milder disease $(2,9)$. Adachi et al. have described an increase in $\mathrm{Na}^{+}$reabsorption from renal tubules in systemic PHA1 as a result of a higher $\mathrm{Na}-\mathrm{Cl}$ cotransporter protein that increases with age. This may be a sensible explanation for the gradual reduction in the requirement of oral salt in our patient as he grew up (8).

The associated episodic hyperlipidemia, in our patient, could not be fully elucidated. Although it has been suggested that dehydration during fasting increases serum lipids and lipoproteins, we think this cannot solely explain the excessive increase of lipids, especially triglyceride, in our patient during the salt-losing crises. In this patient, the serum triglyceride levels approached three times the normal range's upper limit. Additionally, samples for lipid profile were taken mainly on the next day of admission, when the patient has received reasonable rehydration. Previous work has elaborated on the relationship between the renin-angiotensin-aldosterone system (RAAS) and lipid metabolism $(11,12,13)$. These studies have described a clear correlation between the serum level of aldosterone and the concentration of lipid metabolic components in the peripheral circulation. Hannemann et al., in an adult population study, have demonstrated a positive association of serum aldosterone level with metabolic syndrome and high serum triglyceride, and a negative association with serum high-density lipoprotein cholesterol (HDL-C) (14). A later similar study by Hannich et al. complemented the picture by showing a positive correlation with low-density lipoprotein cholesterol (LDL-C) (12). Despite not providing proof for a causal relationship, these two studies, in addition to earlier smaller studies, make these relationships between plasma aldosteronelevel and HDL-Calmost unchallengeable $(15,16,17)$. If this is the situation, then the episodes of dyslipidemia in our patient, which roughly coincided with the salt-wasting events and improved with treatment, could be attributable to the activated RAAS and the resultant high aldosterone level. Whenever the intravascular fluid volume is replenished, and hyperkalemia and hyponatremia are corrected, the stimulus for RAAS activation is lost or reduced. The expected drop in serum aldosterone level can then explain why the lipid profile returned to normal in-between 
the crises in our patient. A proper follow-up, particularly for the lipid profile, insulin resistance, and other components of the metabolic syndrome, should be undertaken in the long-term in our patient. None of the reviewed case reports and case series of systemic PHA1 has depicted a similar finding. However, lipid profile might merely have not been tested in those cases. The incidental finding of a lipemic blood sample prompted us to verify the lipid status of our patient. Further future observations are needed to answer whether the finding of hypertriglyceridemia is peculiar to our patient's genetic syndrome or is a more widespread under-reported phenomenon in similar cases. Until then, this finding's potential utility in the clinical setting, for example, as a differential diagnostic tool or to prevent possible sequelae such as secondary pancreatitis, will remain not clearly defined.

Regarding the papular skin rash, it has been wellreported in the literature that patients with systemic PHA1 are more prone to skin infections and rash flare-ups that usually precede severe salt-wasting crises. The secretion of sweat with elevated sodium chloride concentrations is thought to directly affect the eccrine ducts (30), and patients may present with different cutaneous features like seborrheic dermatitis, folliculitis, or miliaria rubralike lesions. Although scabies has not been specifically reported with systemic PHA1, it can still be explained by the mechanism aforementioned. Any rash flare-up in PHA1 should alert physicians and families about impending saltwasting crises to take preventive measures.

Mutations involving ENaC $\beta$ subunits and the published cases with a missense mutation, as in our patient, are even rarer than those with $\alpha$ subunit mutations and non-missense mutations, respectively. In the other two Sudanese children in the literature, one had an $\alpha$ mutation, while no mutation was revealed in the other $(9,10)$. The homozygous missense variant found in our patient affects a highly conserved amino acid, it was predicted to be pathogenic by all applied in silico programs (SIFT, Mutation Taster, Align GVGD, and Poplyphen), no homozygotes were detected for this variant, and the allele frequency of this variant was $2.121 \times 10^{5}$ in the Genome Aggregation Database (gnomAD) Browser.

\section{Declaration of interest}

The authors declare that there is no conflict of interest that could be perceived as prejudicing the impartiality of the research reported.

\section{Funding}

This research did not receive any specific grant from any funding agency in the public, commercial or not-for-profit sector.
Patient consent

An informed consent was obtained from the patient's guardian.

\section{Author contribution statement}

$A$ A, R T, and A S wrote the case report. A A and R T contributed to writing of the discussion. M Alhassan and M Abdullah wrote most of the discussion. $\mathrm{H}$ Tawamie did the genetic testing and wrote the relevant text. All authors, except H T oversaw the patient's management process.

\section{Acknowledgements}

M A would like to thank the Deanship of Scientific Research at Prince Sattam Bin Abdulaziz University for technically supporting this work. The authors would like to thank Professor Alan D. Rogol, MD - University of Virginia, for critically revising the report.

\section{References}

1 Tajima T, Morikawa S \& Nakamura A. Clinical features and molecular basis of pseudohypoaldosteronism type 1. Clinical Pediatric Endocrinology 201726 109-117. (https://doi.org/10.1297/cpe.26.109)

2 Edelheit O, Hanukoglu I, Gizewska M, Kandemir N, TenenbaumRakover Y, Yurdakök M, Zajaczek S \& Hanukoglu A. Novel mutations in epithelial sodium channel $(\mathrm{ENaC})$ subunit genes and phenotypic expression of multisystem pseudohypoaldosteronism. Clinical Endocrinology 200562 547-553. (https://doi.org/10.1111/j.13652265.2005.02255.x)

3 Adachi M, Tachibana K, Asakura Y, Abe S, Nakae J, Tajima T \& Fujieda K. Compound heterozygous mutations in the $\gamma$ subunit gene of ENaC (1627delG and 1570-1G $\rightarrow$ A) in one sporadic Japanese patient with a systemic form of pseudohypoaldosteronism Type 1. Journal of Clinical Endocrinology and Metabolism 200186 9-12. (https://doi. org/10.1210/jcem.86.1.7116)

4 Amin N, Alvi NS, Barth JH, Field HP, Finlay E, Tyerman K, Frazer S, Savill G, Wright NP, Makaya T, et al. Pseudohypoaldosteronism type 1: clinical features and management in infancy. Endocrinology, Diabetes and Metabolism Case Reports 20132013 130010. (https://doi. org/10.1530/EDM-13-0010)

5 Kerem E, Bistritzer T, Hanukoglu A, Hofmann T, Zhou Z, Bennett W, MacLaughlin E, Barker P, Nash M, Quittell L, et al. Pulmonary epithelial sodium-channel dysfunction and excess airway liquid in pseudohypoaldosteronism. New England Journal of Medicine 1999341 156-162. (https://doi.org/10.1056/NEJM199907153410304)

6 Bizzarri C, Pedicelli S, Cappa M \& Cianfarani S. Water balance and 'salt wasting' in the first year of life: the role of aldosterone-signaling defects. Hormone Research in Paediatrics 201686 143-153. (https://doi. org/10.1159/000449057)

7 Sharma R, Pandey M, Kanwal SK \& Zennaro MC. Pseudohypoaldosteronism type 1: management issues. Indian Pediatrics 201350 331-333. (https://doi.org/10.1007/s13312-013-0070-8)

8 Adachi M, Asakura Y, Muroya K, Tajima T, Fujieda K, Kuribayashi E \& Uchida S. Increased Na reabsorption via the Na-Cl cotransporter in autosomal recessive pseudohypoaldosteronism. Clinical and Experimental Nephrology 2010 14 228-232. (https://doi.org/10.1007/s10157-010-0277-0)

9 Welzel M, Akin L, Büscher A, Güran T, Hauffa BP, Högler W, Leonards J, Karges B, Kentrup H, Kirel B, et al. Five novel mutations in the SCNN1A gene causing autosomal recessive pseudohypoaldosteronism type 1. European Journal of Endocrinology 2013168 707-715. (https:// doi.org/10.1530/EJE-12-1000)

10 Chang SS, Grunder S, Hanukoglu A, Rösler A, Mathew PM, Hanukoglu I, Schild L, Lu Y, Shimkets RA, Nelson-Williams C, et al. Mutations in 
subunits of the epithelial sodium channel cause salt wasting with hyperkalaemic acidosis, pseudohypoaldosteronism type 1 . Nature Genetics 199612 248-253. (https://doi.org/10.1038/ng0396-248)

11 Strazzullo P \& Galletti F. Impact of the renin-angiotensin system on lipid and carbohydrate metabolism. Current Opinion in Nephrology and Hypertension 200413 325-332. (https://doi.org/10.1097/00041552200405000-00010)

12 Hannich M, Wallaschofski H, Nauck M, Reincke M, Adolf C, Völzke H, Rettig R \& Hannemann A. Physiological aldosterone concentrations are associated with alterations of lipid metabolism: observations from the general population. International Journal of Endocrinology 2018 2018 4128174. (https://doi.org/10.1155/2018/4128174)

13 Krug AW \& Ehrhart-Bornstein M. Aldosterone and metabolic syndrome: is increased aldosterone in metabolic syndrome patients an additional risk factor? Hypertension 200851 1252-1258. (https://doi. org/10.1161/HYPERTENSIONAHA.107.109439)

14 Hannemann A, Meisinger C, Bidlingmaier M, Döring A, Thorand B, Heier M, Belcredi P, Ladwig KH, Wallaschofski H, Friedrich N, et al.
Association of plasma aldosterone with the metabolic syndrome in two German populations. European Journal of Endocrinology 2011164 751-758. (https://doi.org/10.1530/EJE-10-1074)

15 Goodfriend TL, Egan B, Stepniakowski K \& Ball DL. Relationships among plasma aldosterone, high-density lipoprotein cholesterol, and insulin in humans. Hypertension 199525 30-36. (https://doi. org/10.1161/01.hyp.25.1.30)

16 Adolf C, Asbach E, Dietz AS, Lang K, Hahner S, Quinkler M, Rump LC, Bidlingmaier M, Treitl M, Ladurner R, et al. Worsening of lipid metabolism after successful treatment of primary aldosteronism. Endocrine 201654 198-205. (https://doi.org/10.1007/s12020-016-0983-9)

17 Musani SK, Vasan RS, Bidulescu A, Liu J, Xanthakis V, Sims M, Gawalapu RK, Samdarshi TE, Steffes M, Taylor HA, et al. Aldosterone, C-reactive protein, and plasma b-type natriuretic peptide are associated with the development of metabolic syndrome and longitudinal changes in metabolic syndrome components: findings from the Jackson heart study. Diabetes Care 201336 3084-3092. (https://doi.org/10.2337/dc12-2562)

Received in final form 26 April 2021

Accepted 25 May 2021 